\title{
High-lying Rydberg states and ionization energy of vinyl chloride studied by two-photon resonant ionization spectroscopy
}

\author{
Jia-lin Chang a , Jau-Chin Shieh ${ }^{\mathrm{b}}$, Jen-Chieh Wu ${ }^{\mathrm{b}}$, Runhua $\mathrm{Li}^{\mathrm{a}}$, Yit-Tsong Chen ${ }^{\mathrm{a}, \mathrm{b}, *}$ \\ ${ }^{\mathrm{a}}$ Institute of Atomic and Molecular Sciences, P.O. Box 23-166, Taipei 106, Taiwan \\ ${ }^{\mathrm{b}}$ Department of Chemistry, National Taiwan University, Taipei 106, Taiwan
}

Received 14 April 2000; in final form 7 June 2000

\begin{abstract}
High-lying Rydberg states of jet-cooled vinyl chloride at 9.3-10 eV are investigated using $2+1$ resonance-enhanced multiphoton ionization spectroscopy. Four Rydberg series are observed and tentatively assigned as due to the transitions of $\pi \rightarrow n \mathrm{~s}, n \mathrm{p} \sigma, n \mathrm{p} \pi$, and $n \mathrm{f}$ Rydberg orbitals associated with quantum defects of $0.94,0.89,0.48$, and 0.02 , respectively. All of the four series converge to the same ionization energy limit corresponding to the ground state of vinyl chloride cation. The adiabatic ionization energy of vinyl chloride is determined to be $80720 \pm 6 \mathrm{~cm}^{-1}$, in excellent agreement with previously reported values. (C) 2000 Elsevier Science B.V. All rights reserved.
\end{abstract}

\section{Introduction}

The spectroscopy [1-9] and photodissociation dynamics [10-17] of vinyl chloride $\left(\mathrm{C}_{2} \mathrm{H}_{3} \mathrm{Cl}\right)$ have been intensively examined in the past decades. Vinyl chloride, being an atmospheric pollutant, is of interest because it has been widely used in the industry for poly(vinyl chloride) production. The understanding of its photochemical processes is therefore very important. While photodissociation experiments usually measure the internal state, velocity, and angular distributions of photofragments, which are closely related to the topological features of the potential

\footnotetext{
* Corresponding author. Academia Sincia, Institute of Atomic and Molecular Sciences, P.O. Box 23-166, Taipei 106, Taiwan. Fax: +886-2-2362-0200; e-mail: ytchen@pub.iams.sinica.edu.tw
}

energy surface of photodissociative molecules, spectroscopic investigation is the most stringent examination for molecular excited states and their corresponding potential energy surfaces.

Walsh reported the vacuum-ultraviolet (VUV) absorption spectrum of vinyl chloride at $105-220 \mathrm{~nm}$ and identified two Rydberg series which converge to $\sim 10 \mathrm{eV}$ [18]. Sood and Watanabe measured the absorption and ionization coefficients of the same molecule in the region of 107.5-200 nm [19]. In addition to the two series observed by Walsh, Sood and Watanabe found two new Rydberg series. Berry recorded the VUV absorption spectra of chloroethylenes up to $70000 \mathrm{~cm}^{-1}$ but without spectroscopic assignment [20]. The early studies on the excited electronic states of chloroethylenes before 1984 have been reviewed by Robin [21]. Recently, Locht et al. reported the absorption spectrum of vinyl chloride at 8-12 eV (103-155 $\mathrm{nm})$ and determined the first 
ionization energy ( $I E$ ) of vinyl chloride to be 10.013 $\pm 0.005 \mathrm{eV}$ [2], in agreement with that obtained from photoelectron spectroscopy [3]. The two-photon resonance-enhanced multiphoton ionization (REMPI) spectroscopy of vinyl chloride was studied first by Williams and Cool in the spectral range of 7.1-9.3 $\mathrm{eV}\left(57100-75000 \mathrm{~cm}^{-1}\right)$ [1]. Many vibronic structures were observed and assigned below 75000 $\mathrm{cm}^{-1}$.

In this Letter, we report the $2+1$ REMPI spectrum of vinyl chloride at 9.3-10 eV (75000-80650 $\mathrm{cm}^{-1}$ ) which is complementary to that obtained by Williams and Cool [1]. We have identified and assigned four Rydberg series of vinyl chloride, all of which converge to the same first $I E$ limit. The adiabatic $I E$ of vinyl chloride determined from this study is $80720 \pm 6 \mathrm{~cm}^{-1}$, in excellent agreement with previously reported values. The $2+1$ REMPI spectrum of vinyl chloride at 7.4-10 eV has been re-examined in our laboratory, and a complete assignment facilitated with calculated Franck-Condon factors by ab initio methods $[22,23]$ will be discussed in a later publication.

\section{Experiment}

The experimental details for obtaining $2+1$ REMPI molecular spectroscopy in our laboratory were described in an earlier publication [24]. Briefly, vinyl chloride was seeded in 2 atm He to form a $10 \%$ mixture and was expanded into a vacuum chamber through a pulsed valve. A focused laser beam crossed the collimated molecular beam and ionized vinyl chloride. The ions were repelled by electric field in a direction perpendicular to both laser and molecular beams. The ions then moved across a field-free time-of-flight (TOF) tube and were finally detected by a microsphere plate. Due to the weak $\mathrm{C}_{2} \mathrm{H}_{3} \mathrm{Cl}^{+}$signal (too week to be seen in Fig. 1), a photon counter was utilized to obtain the REMPI spectrum of vinyl chloride when gated on this parent ion $(m / z=62)$. Alternatively, the signal of the $m / z=27$ mass channel $\left(\mathrm{C}_{2} \mathrm{H}_{3}^{+}\right)$, the strongest in the TOF observation (Fig. 1), was monitored as a function of laser wavelength for better signal-to-noise ratio. Care was taken to insure that the REMPI

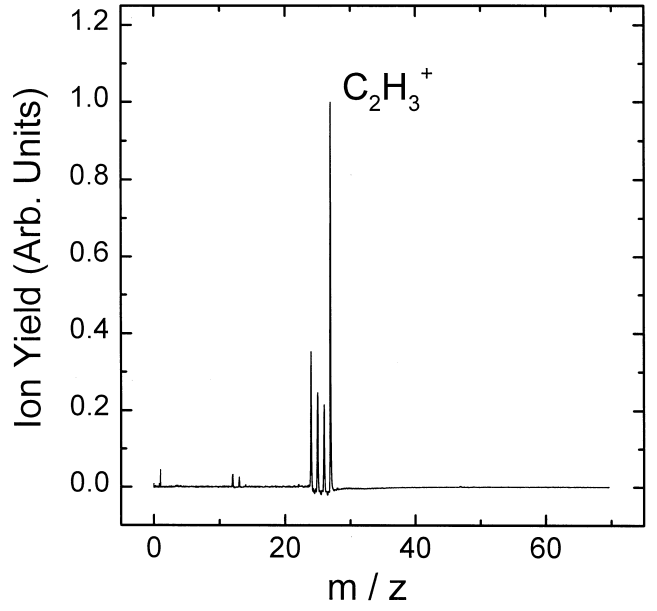

Fig. 1. Typical TOF mass spectrum of vinyl chloride excited at $250 \mathrm{~nm}$ via $2+1$ REMPI.

spectra are identical by monitoring either the fragmentary $\mathrm{C}_{2} \mathrm{H}_{3}^{+}$or the parent $\mathrm{C}_{2} \mathrm{H}_{3} \mathrm{Cl}^{+}$. Optogalvanic spectroscopy of $\mathrm{Ne}$ was employed to calibrate the laser wavelength.

The polarization-ratio of vinyl chloride in a twophoton absorption process was also measured. The polarization-ratio, $\Omega$, is defined as the band-intensity ratio for circularly to linearly polarized radiations. For the polarization experiments, a linear polarizer was used to form linearly polarized laser radiation. The linearly polarized laser then passes through a double Fresnel rhomb and a Fresnel rhomb, functioning respectively as half-wave and quarter-wave retarders. By rotating the double Fresnel rhomb, the polarization vector of the laser is set at $0^{\circ} / 45^{\circ}$ with respect to the optical axis of the Fresnel rhomb to form linearly/circularly polarized radiation. Further details for the quantitative determination of polarization-ratios can be found in Ref. [24].

\section{Results and discussion}

Fig. 2 shows the REMPI spectrum of vinyl chloride at $76000-80750 \mathrm{~cm}^{-1}$. The positions of sharp and well-resolved peaks are tabulated in Table 1, while the peaks with very weak intensity or congested with other bands are listed in parentheses. The dramatic rise on the right side of Fig. 2 at $\sim 80600$ 


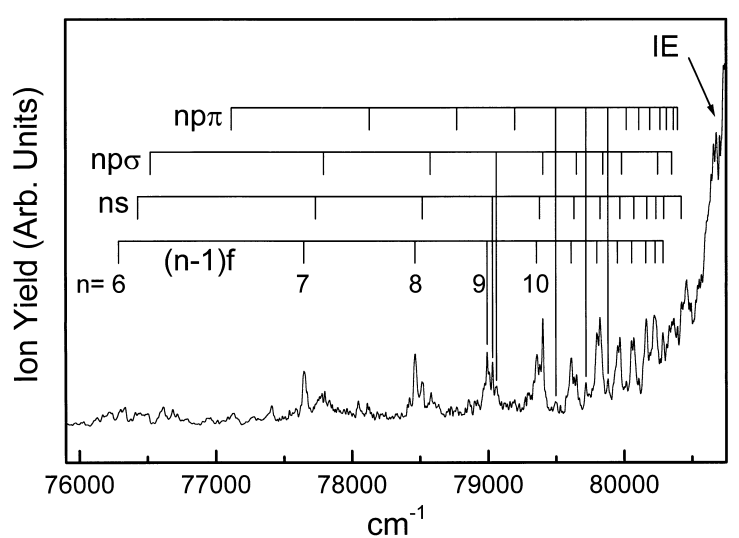

Fig. 2. Composite $2+1$ REMPI spectrum of vinyl chloride at 9.4-10 eV.

$\mathrm{cm}^{-1}$ marks the onset of the ionization continuum of vinyl chloride. The observed transitions are analyzed using the Rydberg formula [25]

$\nu=I E-R /(n-\delta)^{2}$

where $\nu$ is the transition frequency in $\mathrm{cm}^{-1}, R=$ $109736.3588 \mathrm{~cm}^{-1}$ is the Rydberg constant taking the mass of vinyl chloride into account, $n$ is a

Table 1

Peak positions $\left(\mathrm{cm}^{-1}\right)$, ionization energies, and quantum defects for the four Rydberg series of vinyl chloride observed at 9.3-10 $\mathrm{eV}^{\mathrm{a}}$

\begin{tabular}{lcccl}
\hline$n$ & $(\pi, n \mathrm{~s})$ & $(\pi, n \mathrm{p} \sigma)$ & $(\pi, n \mathrm{p} \pi)$ & $(\pi, n \mathrm{f})$ \\
\hline 5 & & & & 76288 \\
6 & $(76428)$ & $(76520)$ & 77112 & 77646 \\
7 & $(77732)$ & 77790 & 78128 & 78464 \\
8 & 78518 & 78575 & $(78770)$ & 78992 \\
9 & 79032 & 79060 & $(79195)$ & 79356 \\
10 & 79379 & 79403 & 79498 & 79611 \\
11 & 79632 & 79650 & 79720 & 79800 \\
12 & 79823 & $(79844)$ & 79881 & 79951 \\
13 & 79970 & $(79983)$ & 80017 & 80056 \\
14 & 80073 & & 80109 & 80160 \\
15 & $(80167)$ & & 80190 & 80229 \\
16 & $(80235)$ & $(80248)$ & $(80265)$ & 80288 \\
17 & $(80293)$ & & 80313 & \\
18 & & 80351 & 80364 & \\
19 & & & 80394 & \\
20 & 80421 & & & \\
$I E$ & $80720 \pm 2$ & $80729 \pm 3$ & $80714 \pm 3$ & $80716 \pm 2$ \\
$\delta$ & $0.94 \pm 0.01$ & $0.89 \pm 0.01$ & $0.48 \pm 0.01$ & $0.02 \pm 0.01$ \\
\hline
\end{tabular}
bands. principal quantum number, and $\delta$ is the corresponding quantum defect. Four Rydberg series have been identified as due to the promotion of a $\pi$-electron to the $n \mathrm{~s}, n \mathrm{p} \sigma, n \mathrm{p} \pi$, and $n \mathrm{f}$ Rydberg orbitals, supported by their $\delta$ values.

\section{1. $(\pi, n s)$ Rydberg series}

The promotion of a $\pi$-electron to the $n$ s Rydberg orbital results in the $(\pi, n \mathrm{~s})$ Rydberg state. Fig. 3 shows the best fit of the $\pi \rightarrow n$ s transitions to the Rydberg formula rendering a converged limit at $80720 \pm 2 \mathrm{~cm}^{-1}$ with $\delta=0.94(n=6-17,20)$. The $\delta$ value of 0.94 is quite typical for an s Rydberg series of hydrocarbon molecules $(\delta \sim 1.0)$ [25]. In order to compare our data with those obtained from one-photon absorption spectra [2,18,19], we have re-examined the transitions reported previously. The $\mathrm{R}_{2}$ series of Walsh [18] ( IE $=80877 \pm 103 \mathrm{~cm}^{-1}$, $\delta=0.94, n=3-9)$ and of Sood and Watanabe [19] $(I E=81008 \pm 177, \quad \delta=0.97, \quad n=3-7,9) \quad$ should correspond to this $(\pi, n \mathrm{~s})$ series. The more accurate $I E=80720 \pm 2 \mathrm{~cm}^{-1}$ value, obtained from the present experiment, is resulted from the higher spectral resolution and the better fitting of the Rydberg states with higher $n$ quantum numbers. It is well known that deviation from the Rydberg formula is amplified when orbitals of smaller $n$ are included in the fitting. The reasoning for that is stemmed from the probable penetration of the low-lying s electrons into molecular core [24,25].

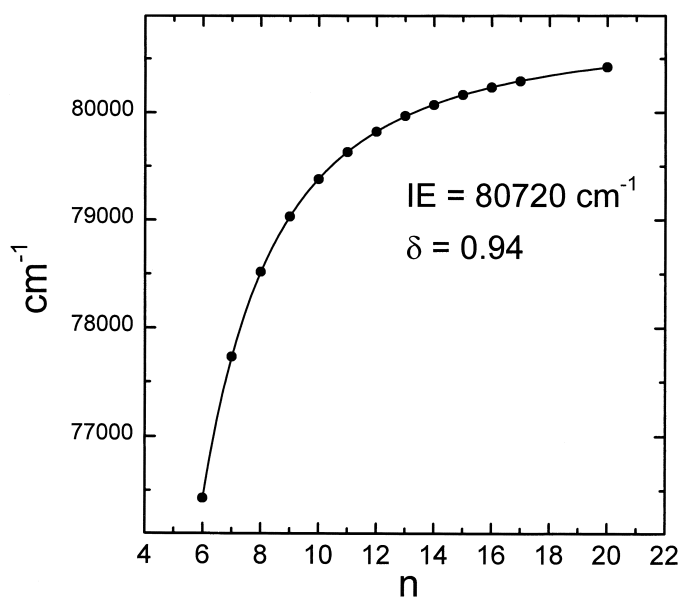

Fig. 3. Best fit of the $(\pi, n s)$ series to the Rydberg formula. 
In a previous study by Locht et al. [2], this s Rydberg series, however, was assigned as an $n \mathrm{~d}$ $(n=3-11,16)$ series with $I E=80756 \pm 38 \mathrm{~cm}^{-1}$ and $\delta=0$. We believe that the $n \mathrm{~d}(\delta=0)$ and $n \mathrm{~s}$ $(\delta=1.0)$ Rydberg series assigned by Locht et al. should be the $(n+1) \mathrm{s}(\delta=1.0)$ and $(n-1) d / \mathrm{f}$ $(\delta=0)$ series, respectively. With this correction, these two series would then be consistent with our assignment of the $\mathrm{ns}(\delta=0.94)$ and the $n \mathrm{f}(\delta=0.02$, see Section 3.4) Rydberg series. In the present study, higher resolution $\left(\sim 2 \mathrm{~cm}^{-1}\right)$ to discern the detailed spectral features and better fitting to the Rydberg formula should give more definitive conclusion. For example, taking a closer look at Fig. 2, we have resolved the peaks of $(n-1) \mathrm{f}, n \mathrm{~s}$ and $n \mathrm{p} \sigma$ series (e.g., 8f, 9s, and 9po), even though they are clustered together. In sharp contrast, the previous low-resolution $\left(\sim 40 \mathrm{~cm}^{-1}\right)$ one-photon absorption spectra $[2,18,19]$ were unable to observe the $n \mathrm{p \sigma}$ Rydberg states at all.

\section{2. $(\pi, n p \sigma)$ Rydberg series}

Vinyl chloride is a planar molecule of $\mathrm{C}_{\mathrm{s}}$ symmetry and therefore possessed of one $n \mathrm{p} \pi$ (out-of-plane) and two $n \mathrm{p} \sigma$ (in-plane) molecular orbitals. By definition, the molecular plane is designated as $y z$-plane. The $n \mathrm{p}_{x}$ thus corresponds to the $n \mathrm{p} \pi$ Rydberg orbital, and the $n \mathrm{p}_{y}$ and $n \mathrm{p}_{z}$ correlate with the npo orbitals. In this study, we have observed two $(\pi, n \mathrm{p})$ series, and attributed them to $(\pi, n \mathrm{p} \sigma)$ and $(\pi, n \mathrm{p} \pi)$. The $(\pi, n \mathrm{p} \sigma)$ series, converging to $80729 \pm 3 \mathrm{~cm}^{-1}$ with $\delta=0.89(n=6-13,16,18)$, is observed for the first time (Table 1). The $(\pi, n \mathrm{p} \pi)$ series will be discussed in Section 3.3.

The quantum defect (0.89) for the $n \mathrm{p} \sigma$ series of vinyl chloride is slightly larger than those for the $3 p_{y}$ (0.76) and $3 \mathrm{p}_{z}$ (0.72) Rydberg states of ethylene $\left(\mathrm{C}_{2} \mathrm{H}_{4}\right)$ [26]. The $n \mathrm{p}_{y}$ and $n \mathrm{p}_{z}(n>3)$ Rydberg states of ethylene and other chloroethylenes have unfortunately not been reported to date for comparison. Comparing with ethylene, the presence of the $\mathrm{Cl}$ atom in $\mathrm{C}_{2} \mathrm{H}_{3} \mathrm{Cl}$ is responsible for the larger $\delta$ value in the $n \mathrm{p} \sigma$ Rydberg series. The $\mathrm{Cl}$ atom, a third-row element, is expected to cause larger quantum defect than the $\mathrm{C}$ (second-row) and $\mathrm{H}$ (first-row) atoms. It is difficult, however, to distinguish the contribution of $\left(\pi, n \mathrm{p}_{y}\right)$ from that of $\left(\pi, n \mathrm{p}_{z}\right)$ at the present study. Consequently, this series is denoted as $(\pi, n \mathrm{p} \sigma)$.

\section{3. $(\pi, n p \pi)$ Rydberg series}

The series converging to $80714 \pm 3 \mathrm{~cm}^{-1}$ with $\delta=0.48(n=6-19)$ is due to the $\pi \rightarrow n \mathrm{p} \pi\left(n \mathrm{p}_{x}\right)$ transition (Table 1; e.g., 10, 11, and 12p $\pi$ in Fig. 2). The observed quantum defect is close to a typical value $(\delta \sim 0.5)$ for the $n \mathrm{p}$ Rydberg states of hydrocarbons [25]. This series corresponds to the $\mathrm{R}_{3}$ series reported by Sood and Watanabe [19] ( $I E=80735 \pm$ $\left.31 \mathrm{~cm}^{-1}, \delta=0.61, n=3-8\right)$ and the $n \mathrm{p}$ series of Locht et al. [2] $\left(I E=80754 \pm 10 \mathrm{~cm}^{-1}, \delta=0.59\right.$, $n=4-14,24)$. Identifying this series as due to $(\pi, n \mathrm{p} \pi)$, rather than $(\pi, n \mathrm{p} \sigma)$, is based on a polarization-ratio measurement.

According to the two-photon theory for a $\mathrm{C}_{\mathrm{s}}$ molecule, $\Omega=3 / 2$ for the transitions from the $\mathrm{A}^{\prime}$ ground state to $\mathrm{A}^{\prime \prime}$ excited electronic states and $\Omega<$ $3 / 2$ for those to the $\mathrm{A}^{\prime}$ states. Within a $\mathrm{C}_{\mathrm{s}}$ group, the $(\pi, n \mathrm{p} \pi)$ Rydberg states of $\mathrm{C}_{2} \mathrm{H}_{3} \mathrm{Cl}$ are of $\mathrm{A}^{\prime}$ symmetry and the $(\pi, n \mathrm{p} \sigma)$ states are $\mathrm{A}^{\prime \prime}$. Our polarization-ratio experiment on this series clearly indicates $\mathrm{A}^{\prime}$ symmetry $(\Omega<0.8)$ for the upper states. The $\mathrm{A}^{\prime}$ symmetry of $(\pi, n \mathrm{p} \pi)$ is also consistent with that of $3 \mathrm{p}_{x}$ and $4 \mathrm{p}_{x}$ Rydberg states reported by Williams and Cool [1]. Accordingly, this series can be unambiguously assigned as the $(\pi, n \mathrm{p} \pi)$ Rydberg states. It is worthwhile to note that the quantum defect (0.89) of the in-plane Rydberg orbitals $(n p \sigma)$ is more pronounced than that $(0.48)$ of the out-of-plane $(n \mathrm{p} \pi)$ orbital, because of the effective contribution of the $\mathrm{Cl}$ atom to the $n \mathrm{p} \sigma$ orbitals in vinyl chloride.

\section{4. $(\pi, n f)$ Rydberg series}

The series converging to $80716 \pm 2 \mathrm{~cm}^{-1}$ with $\delta=0.02(n=5-16)$, obtained in this study, is $(\pi, n \mathrm{f})$ Rydberg series, and is the same $\mathrm{R}_{1}$ series as Walsh [18] ( $I E=80648 \pm 6, \delta=0.04, n=4-11)$ and Sood and Watanabe [19] $\left(I E=80656 \pm 16 \mathrm{~cm}^{-1}, \delta=\right.$ $0.04, n=4-9)$ reported. The ns Rydberg series assigned by Locht et al. [2] should be the $n \mathrm{f}$ series $\left(I E=80754 \pm 10 \mathrm{~cm}^{-1}, \quad \delta=0.09, \quad n=4-9\right) \quad$ as 
mentioned earlier. This series is assigned as $(\pi, n f)$, rather than $(\pi, n \mathrm{~d})$, based on the following examinations. First, no $3 \mathrm{~d}$ signals with $\delta \sim 0.02$ were observed in the REMPI spectra obtained by us and by Williams and Cool [1]. Second, the observed series starts with $n=4$ (a weak transition at $\sim 73700$ $\mathrm{cm}^{-1}$, not listed in Table 1$)$, and the very small $\delta$ value is also favorable to the $(\pi, n \mathrm{f})$ assignment. Furthermore, the $n=3$ Rydberg members observed by Walsh [18] $\left(67979 \mathrm{~cm}^{-1}\right)$, Sood and Watanabe [19] $\left(68000 \mathrm{~cm}^{-1}\right)$, and Loch et al. [2] $(8.498 \mathrm{eV}$, $68543 \mathrm{~cm}^{-1}$ ) are probably due to the $n_{\mathrm{Cl}} \rightarrow 3 \mathrm{~s}$ transition reported by Williams and Cool [1] (67904 and $\left.68608 \mathrm{~cm}^{-1}\right)$. In Fig. 2, sharp spectral features for the $(\pi, n \mathrm{f})$ Rydberg series of vinyl chloride are shown, similar to those observed in ethylene [26] and other chloroethylenes [1,27].

\subsection{Adiabatic IE}

The four identified Rydberg series fit the Rydberg formula very well with uncertainty of only a few $\mathrm{cm}^{-1}$ (Table 1). The four Rydberg series all converge to the same ionization limit, corresponding to the ${ }^{2} \mathrm{~A}^{\prime \prime}$ ground state of $\mathrm{C}_{2} \mathrm{H}_{3} \mathrm{Cl}^{+}$. We have taken the average $I E$ value of the four Rydberg series, 80720 $\pm 6 \mathrm{~cm}^{-1}(10.0080 \pm 0.0007 \mathrm{eV})$, as the adiabatic $I E$ of vinyl chloride (Table 1). Our adiabatic $I E$ is in excellent agreement with previous values obtained from different methods, such as VUV absorption [2] $(10.013 \pm 0.005 \mathrm{eV})$, photoelectron [3] $(10.00 \pm 0.1$ $\mathrm{eV})$, and photoionization spectroscopy [4] (9.98 \pm $0.02 \mathrm{eV})$.

\section{Conclusion}

We have observed and assigned four Rydberg series of vinyl chloride at 9.3-10 eV using $2+1$ REMPI spectroscopy. Identification for the new $(\pi, n \mathrm{p} \sigma)$ Rydberg series and reassignment for the others, $(\pi, n \mathrm{~s}),(\pi, n \mathrm{p} \pi)$, and $(\pi, n \mathrm{f})$, have been made. The adiabatic ionization energy of vinyl chloride is determined to be $80720 \pm 6 \mathrm{~cm}^{-1}$, in agreement with previously reported values, nevertheless with better accuracy. With the aid of calculated
Franck-Condon factors by ab initio methods, a comprehensive analysis for the vibronic spectrum of vinyl chloride at $6-10 \mathrm{eV}$ is underway, and will be discussed in a later publication.

\section{Acknowledgements}

This work is supported by National Science Council of ROC (Grants No. 89-2113-M-001-032) and by China Petroleum. J.L.C. is grateful to Academic Sinica for his postdoctoral fellowship at IAMS.

\section{References}

[1] B.A. Williams, T.A. Cool, J. Phys. Chem. 97 (1993) 1270.

[2] R. Locht, B. Leyh, K. Hottmann, H. Baumgärtel, Chem. Phys. 220 (1997) 207.

[3] R. Locht, B. Leyh, K. Hottmann, H. Baumgärtel, Chem. Phys. 220 (1997) 217.

[4] L. Sheng, F. Qi, L. Tao, Y. Zhang, S. Yu, C.-K. Wong, W.-K. Li, Int. J. Mass Spectrom. Ion Proc. 148 (1995) 179.

[5] K.H. Sze, C.E. Brion, A. Katrib, B. El-Issa, Chem. Phys. 137 (1989) 369.

[6] M. Hayashi, T. Inagusa, J. Mol. Struct. 220 (1990) 103.

[7] I. Merke, L. Poteau, G. Wlodarczak, A. Bouddou, J. Demaison, J. Mol. Spectrosc. 177 (1996) 232.

[8] P. Stoppa, S. Giorgianni, S. Ghersetti, Mol. Phys. 91 (1997) 215.

[9] A. de Lorenzi, S. Giorgianni, R. Bini, Mol. Phys. 96 (1999) 101.

[10] P.T.A. Reilly, Y. Xie, R.J. Gordon, Chem. Phys. Lett. 178 (1991) 511

[11] Y. Mo, K. Tonokura, Y. Matsumi, M. Kawasaki, T. Sato, T. Arikawa, P.T.A. Reilly, Y. Xie, Y.-A. Yang, Y. Huang, R.J. Gordon, J. Chem. Phys. 97 (1992) 4815.

[12] Y. Huang, Y.-A. Yang, G.-X. He, R.J. Gordon, J. Chem. Phys. 99 (1993) 2752.

[13] G. He, Y. Yang, Y. Huang, S. Hashimoto, R.J. Gordon, J. Chem. Phys. 103 (1995) 5488.

[14] P.W. Browning, D.C. Kitchen, M.F. Arendt, L.J. Butler, J. Phys. Chem. 100 (1996) 7765.

[15] K. Tonokura, L.B. Daniels, T. Suzuki, K. Yamashita, J. Phys. Chem. A 101 (1997) 7754.

[16] D.A. Blank, W. Sun, A.G. Suits, Y.T. Lee, S.W. North, G.E. Hall, J. Chem. Phys. 108 (1998) 5414.

[17] P. Farmanara, V. Stert, W. Radloff, Chem. Phys. Lett. 288 (1998) 518

[18] A.D. Walsh, Trans. Faraday Soc. 41 (1945) 35.

[19] S.P. Sood, K. Watanabe, J. Chem. Phys. 45 (1966) 2913.

[20] M.J. Berry, J. Chem. Phys. 61 (1974) 3114. 
[21] M.B. Robin, Higher Excited States of Polyatomic Molecules, vol. 3, Academic, New York, 1985.

[22] A.M. Mebel, Y.-T. Chen, S.H. Lin, Chem. Phys. Lett. 258 (1996) 53.

[23] A.M. Mebel, Y.-T. Chen, S.H. Lin, J. Chem. Phys. 105 (1996) 9007.
[24] J.-C. Shieh, J.-L. Chang, J.-C. Wu, R. Li, A.M. Mebel, N.C. Handy, Y.-T. Chen, J. Chem. Phys. 112 (2000) 7384.

[25] A.B.F. Duncan, Rydberg Series in Atoms and Molecules, Academic, New York, 1971.

[26] B.A. Williams, T.A. Cool, J. Chem. Phys. 94 (1991) 6358.

[27] B.A. Williams, T.A. Cool, J. Chem. Phys. 93 (1990) 1521. 